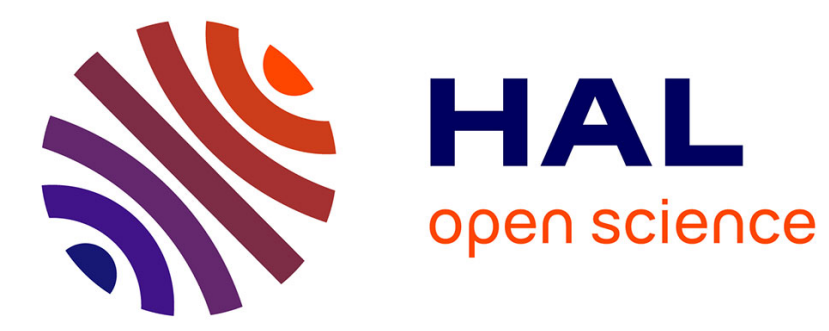

\title{
Existence results for a monophasic compressible Darcy-Brinkman's flow in porous media
}

\author{
Houssein Nasser El Dine, Mazen Saad, Raafat Talhouk
}

\section{To cite this version:}

Houssein Nasser El Dine, Mazen Saad, Raafat Talhouk. Existence results for a monophasic compressible Darcy-Brinkman's flow in porous media. 2017. hal-01558770

\author{
HAL Id: hal-01558770 \\ https://hal.science/hal-01558770
}

Preprint submitted on 10 Jul 2017

HAL is a multi-disciplinary open access archive for the deposit and dissemination of scientific research documents, whether they are published or not. The documents may come from teaching and research institutions in France or abroad, or from public or private research centers.
L'archive ouverte pluridisciplinaire HAL, est destinée au dépôt et à la diffusion de documents scientifiques de niveau recherche, publiés ou non, émanant des établissements d'enseignement et de recherche français ou étrangers, des laboratoires publics ou privés. 


\title{
Existence results for a monophasic compressible Darcy-Brinkman's flow in porous media
}

\author{
Houssein NASSER EL DINE ${ }^{(1,2)}$, Mazen SAAD ${ }^{(1)}$, Raafat TALHOUK ${ }^{(2)}$
}

\begin{abstract}
In this paper, we are interested in the displacement of a single compressible phase in DarcyBrinkman's flow in porous media. The equations are obtained by the conservation of mass and by considering the Brinkman regularization velocity of the standard Darcy infiltration velocity. This model is treated in its general form with the whole nonlinear terms. In first part, we prove the existence in one dimensional space of a solution for the Dracy-Brinkman system, and in the second one we treat this system with Bear hypothesis in multidimensional spaces.
\end{abstract}

\section{Introduction and the Darcy-Brinkman model}

\subsection{Introduction}

Flow in porous media occurs in a range of engineering applications, e.g., geothermal systems, oil extraction, ground water pollution, storage of nuclear waste, heat exchangers, catalytic convertors, and chemical reactors. Among the important characteristics of such flows are the velocity profile and pressure drop [11]. For example, these two directly influence convection heat transfer in porous media, and the required pumping power in heat exchange processes. In convection heat transfer, the velocity profile is substituted into the energy equation to obtain the temperature distribution. In reactors, the velocity profile strongly impacts the chemical reactions (see $[2,4,8,20])$.

Different empirical laws are used to describe the filtration of a fluid through porous media [19]. We mention the Darcy law which states that the filtration velocity of the fluid is proportional to the pressure gradient. The Darcy law cannot sustain the no-slip condition on an impermeable wall or a transmission condition on the contact with free flow. That motivated H. Brinkmann in 1947 to modify the Darcy law in order to be able to impose the no-slip boundary condition on an obstacle submerged in porous medium. He assumed large permeability to compare his law with experimental data and assumed that the second viscosity $\mu$ equals the physical viscosity of the fluid in the case of monophasic flow. Also, an up-scaling of the Stokes equations with non-slip boundary condition describing the flow in a porous medium, leads to the Darcy-Brinkman equations [5].

(1)- École Centrale de Nantes. Laboratoire de Mathématiques Jean Leray. UMR 6629 CNRS, F-44321, Nantes, France

(2)- Lebanese University, Faculty of Sciences and Doctoral School of Sciences and Technology (EDST), Laboratory of Mathematics, Hadath-lebanon 


\subsection{The Darcy-Brinkman model}

We are interested in the displacement of compressible phase in a Darcy-Brinkman flow in an isotropic porous media. The equation describing the displacement of compressible fluid is given by the following mass conservation of the phase:

$$
\phi \partial_{t} \rho(p)+\operatorname{div}(\rho(p) V)=0
$$

where $\rho$ is the density of the fluid which is a given function depend on the pressure $p$ and $\phi$ is the porosity. The velocity $V$ is given by the Brinkman-Darcy law [7,19] :

$$
-v k \Delta V+\tilde{\mu} V=-k \nabla p
$$

where $k$ is the permeability of the porous media, $\tilde{\mu}$ is the usual Darcy viscosity and $v$ is the Brinkman viscosity. It is well known that solutions of hyperbolic conservation laws can develop discontinuities, even for smooth initial data (see, e.g., [13]). The presence of these discontinuities or shock waves implies that solutions of conservation laws are sought in a weak sense and are augmented with additional admissibility criteria or entropy conditions in order to ensure uniqueness. As the phase flow equation (1) involves a conservation law, we need to define a suitable concept of entropy solutions for these equations and show that these solutions are well posed. The main challenge in showing existence is the fact that the velocity field $V$ acts as a coefficient in this equation. Although conservation laws with coefficients have been studied extensively in recent years (see $[1,3,9,10,12,16])$ and references therein, the state of the art results require that the coefficient is a function of bounded variation. However, the fact that the coefficient ( the velocity) depends on $p$ poses a big difficulty to treat our equation as a conservation law equation. In order to obtain a system be resolved, first we develop equation (1), then we divided by $\rho(p)$ to get

$$
\phi \partial_{t} u+V \cdot \nabla u+\operatorname{div} V=0
$$

where $u=\ln (\rho(p))$, that give the pressure $p=\rho^{-1}\left(e^{u}\right)$. In what follows let us define the function $h(z)=$ $\rho^{-1}\left(e^{z}\right)$ and suppose $h$ is regular enough (see paragraph 3.1 for some given examples of $h$ ) which leads us to obtain the general form of Brinkman-Darcy system of a single phase :

$$
\left\{\begin{aligned}
\phi \partial_{t} u+V \cdot \nabla u+\operatorname{div} V & =0 \\
-v k \Delta V+\tilde{\mu} V & =-k \nabla h(u) .
\end{aligned}\right.
$$

The system (4) is a coupled transport-elliptic system (of Hyperbolic-elliptic type) strongly nonlinear (due to the fact that V depends on $\mathrm{u}$ ). The study of such system in PDE is not standard or trivial. To our knowledge, there is not any results or study concerning this system. So our study of this system will be divided into two parts, the first one concerns the system (4) in one-dimensional space and in whole $\mathbb{R}$. The second part concerns the system (4) under the Bear hypothesis in a bounded domain $\Omega \subset \mathbb{R}^{d}$.

First of all we will start by transforming the first equation of (4) into a dispersive nonlinear equation by using the linear elliptic structure of the second equation which allows us to work in a fractional spaces with high regularity. For that, apply formally the Helmholtz operator $(-v k \Delta+\tilde{\mu})$ to the first equation in (4) to get

$$
\left\{\begin{aligned}
\phi \tilde{\mu} \partial_{t} u-\phi v k \operatorname{div}\left(\nabla \partial_{t} u\right)-k \nabla h(u) \cdot \nabla u-2 v k \nabla V: \nabla \nabla u-v k V \cdot \nabla \Delta u-k \operatorname{div}(\nabla h(u)) & =0 \\
-v k \nabla^{2} V+\tilde{\mu} V & =-k \nabla h(u) .
\end{aligned}\right.
$$

We precise the notation that : $\nabla V=(\nabla V)_{i j}=\left(\partial_{j} V_{i}\right)_{i j}, \nabla \nabla u=\left(\partial_{j} \partial_{i} u\right)_{i j}, \nabla^{2} V=\nabla \cdot \nabla V$ and $A: B=\sum_{i j} a_{i j} b_{i j}$

In whats follow, we will solve this system in one dimensional space $\mathbb{R}$, and later we will pose Bear hypothesis on the system (4) in order to solve this system in high dimensional bounded space $\Omega$. 


\subsection{Organization of the paper}

We start by defining some notations in Section 2.1; next in Section 2.2 we show the well-posedness of the Darcy-Brinkman equation in 1D by giving some preliminary result and make some linear analysis to state the main theorem. Then in section 3 we rewrite the Darcy-Brinkman system with Bear hypothesis and state some particular cases of state law. Finally in section 3.2 we prove the existence of a weak solution of a generalized system covering those particular cases.

\section{Study of Darcy-Brinkman model in one dimensional space}

\subsection{Notation}

We denote by $C\left(\lambda_{1}, \lambda_{2}, \ldots\right)$ a constant depending on the parameters $\lambda_{1}, \lambda_{2}, \ldots$. The notation $a \lesssim b$ means that $a \leq C b$, for some nonnegative constant $C$ whose exact expression is of no importance. Let $p$ be any constant with $1 \leq p<\infty$ and denote $L^{p}=L^{p}(\mathbb{R})$ the space of all Lebesgue-measurable functions $f$ with the standard norm

$$
|f|_{L^{p}}=\left(\int_{\mathbb{R}}|f(x)|^{p} d x\right)^{\frac{1}{p}}<\infty .
$$

When $p=2$, we denote the norm $|\cdot|_{L^{2}}$ simply by $|\cdot|_{2}$. The inner product of any functions $f_{1}$ and $f_{2}$ in the Hilbert space $L^{2}(\mathbb{R})$ is denoted by

$$
\left(f_{1}, f_{2}\right)=\int_{\mathbb{R}} f_{1}(x) f_{2}(x) d x
$$

The space $L^{\infty}=L^{\infty}(\mathbb{R})$ consists of all essentially bounded, Lebesgue-measurable function $f$ with the norm

$$
|f|_{L^{\infty}}=\operatorname{ess} \sup |f(x)|<\infty \text {. }
$$

We denote by $W^{1, \infty}=W^{1, \infty}(\mathbb{R})=\left\{f \in L^{\infty}, \partial_{x} f \in L^{\infty}\right\}$ endowed with its canonical norm.

For any real constant $s, H^{s}=H^{s}(\mathbb{R})$ denotes the Sobolev space of all tempered distributions $f$ with the norm $|f|_{H^{s}}=\left|\Lambda^{s} f\right|_{2}<\infty$, where $\Lambda$ is the pseudo-differential operator $\Lambda=\left(1-\partial_{x}^{2}\right)^{\frac{1}{2}}$.

For any functions $u=u(x, t)$ and $v(x, t)$ defined on $[0, T) \times \mathbb{R}$ with $T>0$, we denote the inner product, the $L^{p}$-norm and especially the $L^{2}$-norm, as well as the Sobolev norm, with respect to the spatial variable $x$, by $(u, v)=(u(, t), v(, t)),|u|_{L^{p}}=|u(t, .)|_{L^{p}},|u|_{L^{2}}=|u(t, .)|_{L^{2}}$, and $|u|_{H^{s}}=|u(t, .)|_{H^{s}}$, respectively. $B_{H^{s}}(0, R)$ is the closed ball of center 0 and radius $R$ in $H^{s}$.

Let $C^{k}(\mathbb{R})$ denote the space of $k$-times continuously differentiable functions and $C_{0}^{\infty}(\mathbb{R})$ denote the space of infinitely differentiable functions, with compact support in $\mathbb{R}$.

For any closed operator $T$ defined on a Banach space $X$ of functions, the commutator $[T, f]$ is defined by $[T, f] g=T(f g)-f T(g)$ with $f, g$ and $f g$ belonging to the domain of $T$.

\subsection{Well-posedness of the Darcy-Brinkman equation in $1 D$}

In one dimensional space, the Darcy-Brinkman equations (5) can be simplified, into

$$
\left\{\begin{aligned}
\partial_{t} u-\mu \partial_{x}^{2} \partial_{t} u-a \partial_{x} h(u) \partial_{x} u-2 \mu \partial_{x} V \partial_{x}^{2} u-\mu V \partial_{x}^{3} u-a \partial_{x}^{2} h(u) & =0 \\
-\mu \partial_{x}^{2} V+V & =-a \partial_{x} h(u),
\end{aligned}\right.
$$


where $h(u)=\rho^{-1}\left(e^{u}\right), a=\frac{k}{\tilde{\mu}}$ and $\mu=\frac{k v}{\tilde{\mu}}$. By change the scale in time, we can consider $\phi=1$ which change nothing in the study of this system. For the sake of simplicity, we write

$$
\mathcal{T}=1-\mu \partial_{x}^{2}
$$

The following lemma gives an important invertibility result on $\mathcal{T}$

Lemma 1. The operator $\mathcal{T}: H^{2}(\mathbb{R}) \longrightarrow L^{2}(\mathbb{R})$ is well defined, one-to-one and onto. In particular we have

$$
\left|\mathcal{T}^{-1} f\right|_{H^{s}}+\sqrt{\mu}\left|\mathcal{T}^{-1} \partial_{x} f\right|_{H^{s}} \lesssim|f|_{H^{s}}, \forall s>0 .
$$

Proof : The proof of this Lemma is classical but for the sake of completeness we reproduce the proof. In order to prove the invertibility of $\mathcal{T}$, let us first remark that the quantity $|v|_{*}$ defined as

$$
|v|_{*}^{2}=|v|_{2}^{2}+\mu\left|\partial_{x} v\right|_{2}^{2}
$$

is equivalent to the $H^{1}(\mathbb{R})$-norm but not uniformly with respect to $\mu \in(0,1)$. We define by $H_{*}^{1}(\mathbb{R})$ the space $H^{1}(\mathbb{R})$ endowed with this norm. The bilinear form:

$$
a(u, v)=(\mathcal{T} u, v)=(u, v)+\mu\left(\partial_{x} u, \partial_{x} v\right)
$$

is obviously continuous on $H_{*}^{1}(\mathbb{R}) \times H_{*}^{1}(\mathbb{R})$. Remarking that $a(u, u)=|u|_{*}^{2}$, in particular, $a$ is coercive on $H_{*}^{1}$. Using the Riesz Theorem, for all $f \in L^{2}(\mathbb{R})$, there exists a unique $u \in H_{*}^{1}(\mathbb{R})$ such that, for all $v \in H_{*}^{1}(\mathbb{R})$

$$
a(u, v)=(f, v)
$$

equivalently, there is a unique solution to the equation

$$
\mathcal{T} u=f
$$

We then get from the definition of $\mathcal{T}$ that $\partial_{x}^{2} u=\frac{1}{\mu}(u-f)$. Since $u \in H^{1}(\mathbb{R})$ and $f \in L^{2}(\mathbb{R})$, we get $\partial_{x}^{2} u \in L^{2}(\mathbb{R})$ and thus $u \in H^{2}(\mathbb{R})$. We prove here estimate (7). Indeed, from equation (8) by using Cauchy-Schwartz inequality we have

$$
a(u, u) \leq|f|_{2}|u|_{2} \leq \frac{1}{2}|f|_{2}^{2}+\frac{1}{2}|u|_{2}^{2} .
$$

Then it is easy to get $|u|_{*}^{2} \lesssim|f|_{2}^{2}$. We prove here that $\left|\mathcal{T}^{-1} f\right|_{H^{s}}+\sqrt{\mu}\left|\mathcal{T}^{-1} \partial_{x} f\right|_{H^{s}} \lesssim|f|_{H^{s}}$. Indeed, if $f \in H^{s}$ and $u=\mathcal{T}^{-1} f$ then $\mathcal{T} u=f$. Applying $\Lambda^{s}$ to this identity, we get $\mathcal{T} \Lambda^{s} u=\Lambda^{s} f$. Since $\Lambda^{s}$ is commute with $\mathcal{T}$ and $\mathcal{T}^{-1}$, one can use estimate $|u|_{*}^{2} \lesssim|f|_{2}^{2}$ to get the result.

\subsection{Linear analysis}

In order to rewrite the first equation of Darcy-Brinkman system (6) $)_{1}$ in a condensed form, let us define the following operator

$$
A[V, u] f=-a \partial_{x} h(u) f-2 \mu \partial_{x} V \partial_{x} f-\mu V \partial_{x}^{2} f-a \partial_{x} h^{\prime}(u) f-a h^{\prime}(u) \partial_{x} f .
$$

The Darcy-Brinkman system (5) can be written after applying $\mathcal{T}^{-1}$ to both sides of the first equation in (5) as

$$
\left\{\begin{aligned}
-\mu \partial_{x}^{2} V+V & =-a \partial_{x} h(u), \\
\partial_{t} u+B[V, u] \partial_{x} u & =0
\end{aligned}\right.
$$


where $B[V, u]=\mathcal{T}^{-1} A[V, u]$. This subsection is devoted to the proof of energy estimates for the following initial value problem around some reference state $\bar{u}$ :

$$
\left\{\begin{aligned}
-\mu \partial_{x}^{2} V+V & =-a \partial_{x} h(\bar{u}), \\
\partial_{t} u+B[V, \bar{u}] \partial_{x} u & =0 \\
u(0, x) & =u_{0}(x)
\end{aligned}\right.
$$

A natural energy of the second equation of system (10) is given by

$$
E^{s}(u)^{2}=\left(\Lambda^{s} u, \mathcal{T} \Lambda^{s} u\right)=|v|_{H^{s}}^{2}+\mu\left|\partial_{x} v\right|_{H^{s}}^{2}, .
$$

Notice that $E^{s}(u)^{2}$ for a fixed $\mu$ is an equivalent norm of the norm of space $H^{s+1}$. We prove now the following proposition:

Proposition 1. Let $s_{0}>\frac{1}{2}, s \geq s_{0}+1$ and $\bar{u} \in C\left([0, T] ; H^{s+1}\right)$. Then for all $u_{0} \in H^{s+1}$ there exists a unique solution $(u, V)$ to (10), In particular we have for all $0 \leq t \leq T, u \in C\left([0, T] ; H^{s+1}\right) \cap C^{1}\left(0, T ; H^{s}\right)$ and $V \in$ $C^{0}\left([0, T] ; H^{s+2}\right)$, with

$$
E^{S}(u(t, x))^{2} \lesssim e^{\lambda t} E^{s}\left(u_{0}(x)\right)^{2} \exp \left(\frac{1}{\lambda}\left(e^{\lambda t}-1\right)\right)
$$

For some $\lambda=\lambda_{T}=2 \sup _{t \in[0, T]}\left(|\bar{u}|_{H^{s+1}}+1\right)$. And also we have

$$
|V|_{H^{s+2}} \leq C\left(|\bar{u}|_{L^{\infty}},|h|_{C^{\infty}}\right)|\bar{u}|_{H^{s+1}} .
$$

Proof: Before establish the proof let us recall here some product as well as commutator estimates in Sobolev spaces.

Lemma 2 (product estimates). Let $s \geq 0$; one has for all $f, g \in H^{s}(\mathbb{R}) \cap L^{\infty}(\mathbb{R})$,

$$
|f g|_{H^{s}} \lesssim|f|_{L^{\infty}}|g|_{H^{s}}+|f|_{H^{s}}|g|_{L^{\infty}} .
$$

If $s \geq s_{0}>\frac{1}{2}$, one deduces thanks to continuous embedding of Sobolev spaces,

$$
|f g|_{H^{s}} \lesssim|f|_{H^{s}}|g|_{H^{s}}
$$

Let $F \in C^{\infty}(\mathbb{R})$ such that $F(0)=0$. If $g \in H^{s}(\mathbb{R}) \cap L^{\infty}(\mathbb{R})$ with $s \geq 0$, one has $F(g) \in H^{s}(\mathbb{R})$ and

$$
|F(g)|_{H^{s}} \leq C\left(|g|_{L^{\infty}},|F|_{C^{\infty}}\right)|g|_{H^{s}} .
$$

Proof: The proof is classical (see [15, 18, 22]).

Lemma 3 (commutator estimates). For any $s \geq 0$, and $\partial_{x} f, g \in H^{s_{0}}(\mathbb{R}) \cap H^{s-1}(\mathbb{R})$, one has

$$
\left|\left[\Lambda^{s}, f\right] g\right|_{L^{2}} \lesssim\left|\partial_{x} f\right|_{H^{s-1}}|g|_{L^{\infty}}+\left|\partial_{x} f\right|_{L^{\infty}}|g|_{H^{s-1}} .
$$

Thanks to continuous embedding of Sobolev spaces, one has for $s \geq s_{0}+1, s_{0}>\frac{1}{2}$,

$$
\left|\left[\Lambda^{s}, f\right] g\right|_{L^{2}} \lesssim\left|\partial_{x} f\right|_{H^{s-1}}|g|_{H^{s-1}} .
$$

Proof: The proof can be found in Kato and Ponce [15].

Proof of proposition 1:Existence of $V$ is classical and we can easily proof the following estimate on $V$

$$
|V|_{H^{s+2}} \leq C\left(|\bar{u}|_{L^{\infty}},|h|_{C^{\infty}}\right)|\bar{u}|_{H^{s+1}},
$$


indeed,

$$
\begin{aligned}
E^{s}(V)^{2} & =\left(\mathcal{T} \Lambda^{s} V, \Lambda^{s} V\right)=-k\left(\Lambda^{s} \partial_{x}(h(\bar{u})), \Lambda^{s} V\right)=-k\left(\Lambda^{s} \partial_{x}(h(\bar{u})-h(0)), \Lambda^{s} V\right) \\
& =k\left(\Lambda^{s}(h(\bar{u})-h(0)), \Lambda^{s} \partial_{x} V\right)
\end{aligned}
$$

furthermore, for all $\gamma>0$ we have

$$
E^{s}(V)^{2}=k\left(\Lambda^{s}(h(\bar{u})-h(0)), \Lambda^{s} \partial_{x} V\right) \lesssim \frac{1}{\gamma}|h(\bar{u})-h(0)|_{H^{s}}+\gamma\left|\partial_{x} V\right|_{H^{s}},
$$

this yields, for $\gamma$ small enough and by Lemma 2 to

$$
E^{s}(V)^{2} \leq C\left(|\bar{u}|_{L^{\infty}},|h|_{C^{\infty}}\right)|\bar{u}|_{H^{s}}
$$

Replace now $s$ by $s+1$, we get the estimate. Furthermore, in order to prove $V \in C\left([0, T] ; H^{s+2}\right)$ we have that for all $u, v \in B_{H^{s}}(0, R), s>s_{0}+1$

$$
|h(u)-h(v)|_{H^{s}} \leq C\left(R,|h|_{C^{\infty}}\right)|u-v|_{H^{s}},
$$

then from the first equation of (10) and the continuity of $\bar{u}$ one can deduce that $V \in C\left([0, T] ; H^{s+2}\right)$.

On the other hand, under the regularity of $\bar{u}$ and $V$, the existence and uniqueness of a solution $u$ of the second equation of (10) can be achieved like in Appendix A [14] if we have estimate (11). We thus focus our attention on the proof of the energy estimate which is also primordial to the solve the non-linear system. For any $\lambda \in \mathbb{R}$ we compute

$$
e^{\lambda t} \partial_{t}\left(e^{-\lambda t} E^{s}(u)^{2}\right)=-\lambda E^{s}(u)^{2}-\partial_{t}\left(E^{s}(u)^{2}\right)
$$

since

$$
E^{s}(u)^{2}=\left(\Lambda^{s} u, \mathcal{T} \Lambda^{s} u\right)
$$

we have

$$
\partial_{t}\left(E^{s}(u)^{2}\right)=2\left(\mathcal{T} \Lambda^{s} \partial_{t} u, \Lambda^{s} u\right)
$$

One gets using the equation (10) that :

$$
\begin{aligned}
\frac{1}{2} e^{\lambda t} \partial_{t}\left(e^{-\lambda t} E^{s}(u)^{2}\right)= & -\frac{\lambda}{2} E^{s}(u)^{2}+a\left(\Lambda^{s}\left(\partial_{x} h(\bar{u}) \partial_{x} u\right), \Lambda^{s} u\right)+2 \mu\left(\Lambda^{s}\left(\partial_{x} V \partial_{x}^{2} u\right), \Lambda^{s} u\right) \\
& +\mu\left(\Lambda^{s}\left(V \partial_{x}^{3} u\right), \Lambda^{s} u\right)+a\left(\Lambda^{s}\left(\partial_{x} h^{\prime}(\bar{u}) \partial_{x} u\right), \Lambda^{s} u\right)+a\left(\Lambda^{s}\left(h^{\prime}(\bar{u}) \partial_{x}^{2} u\right), \Lambda^{s} u\right) .
\end{aligned}
$$

For all skew-operator $T$ (That is $T^{*}=-T$ ), and for all b smooth enough, one has

$$
\left(\Lambda^{s}(b T u), \Lambda^{s} u\right)=\left(\left[\Lambda^{s}, b\right] T u, \Lambda^{s} u\right)-\frac{1}{2}\left([T, b] \Lambda^{s} u, \Lambda^{s} u\right),
$$

we deduce applying this identity with $\left(T=\partial_{x}\right.$ and $\left.T=\partial_{x}^{3}\right)$ and integrating by parts, 
Existence results for a monophasic compressible Darcy-Brinkman's flow in porous media

$$
\begin{aligned}
& \frac{1}{2} e^{\lambda t} \partial_{t}\left(e^{-\lambda t} E^{s}(u)^{2}\right)=-\frac{\lambda}{2} E^{s}(u)^{2}+a\left(\left[\Lambda^{s}, \partial_{x} h(\bar{u})\right] \partial_{x} u, \Lambda^{s} u\right)-\frac{a}{2}\left(\partial_{x}^{2} h(\bar{u}) \Lambda^{s} u, \Lambda^{s} u\right)+2 \mu\left(\Lambda^{s}\left(\partial_{x} V \partial_{x}^{2} u\right), \Lambda^{s} u\right) \\
&+\mu\left(\left[\Lambda^{s}, V\right] \partial_{x}^{3} u, \Lambda^{s} u\right)-\frac{\mu}{2}\left(\partial_{x}^{3} V \Lambda^{s} u, \Lambda^{s} u\right)-\frac{3 \mu}{2}\left(\partial_{x}^{2} V \partial_{x} \Lambda^{s} u, \Lambda^{s} u\right)-\frac{3 \mu}{2}\left(\partial_{x} V \partial_{x}^{2} \Lambda^{s} u, \Lambda^{s} u\right) \\
&+a\left(\left[\Lambda^{s}, \partial_{x} h^{\prime}(\bar{u})\right] \partial_{x} u, \Lambda^{s} u\right)-\frac{a}{2}\left(\partial_{x}^{2} h^{\prime}(\bar{u}) \Lambda^{s} u, \Lambda^{s} u\right)+a\left(\Lambda^{s}\left(h^{\prime}(\bar{u}) \partial_{x}^{2} u\right), \Lambda^{s} u\right)
\end{aligned}
$$

We now turn to bounding from above the different components of the r.h.s. of (15).

- By using the commutator estimate one gets for all $s>\frac{3}{2}$

$$
a\left(\left[\Lambda^{s}, \partial_{x} h(\bar{u})\right] \partial_{x} u, \Lambda^{s} u\right) \leq a\left|\left[\Lambda^{s}, \partial_{x} h(\bar{u})\right] \partial_{x} u\right|_{2}|u|_{H^{s}} \lesssim\left|\partial_{x}^{2} h(\bar{u})\right|_{H^{s-1}}\left|\partial_{x} u\right|_{H^{s-1}}|u|_{H^{s}} \lesssim\left|\partial_{x}^{2} h(\bar{u})\right|_{H^{s-1}}|u|_{H^{s}}^{2},
$$

then, If we apply Lemma 2 on $F(\bar{u})=h(\bar{u})-h(0)$ we get

$$
a\left(\left[\Lambda^{s}, \partial_{x} h(\bar{u})\right] \partial_{x} u, \Lambda^{s} u\right) \lesssim\left|\partial_{x}^{2} h(\bar{u})\right|_{H^{s-1}}|u|_{H^{s}}^{2} \lesssim|\bar{u}|_{H^{s+1}}|u|_{H^{s}}^{2}
$$

- Thanks to continuous embedding of $H^{s_{0}}(\mathbb{R})$ in $L^{\infty}(\mathbb{R})$ for all $s_{0}>\frac{1}{2}$ we have

$$
\frac{a}{2}\left(\partial_{x}^{2} h(\bar{u}) \Lambda^{s} u, \Lambda^{s} u\right) \lesssim\left|\partial_{x}^{2} h(\bar{u})\right|_{L^{\infty}}|u|_{H^{s}}^{2} \lesssim\left|\partial_{x}^{2} h(\bar{u})\right|_{H^{s}}|u|_{H^{s}}^{2} \lesssim|\bar{u}|_{H^{s_{0}+2}}|u|_{H^{s}}^{2}
$$

- The product estimate give as for all $s \geq s_{0}$, such that $s_{0}>\frac{1}{2}$

$$
\begin{aligned}
2 \mu\left(\Lambda^{s}\left(\partial_{x} V \partial_{x}^{2} u\right), \Lambda^{s} u\right) & =2 \mu\left(\left[\Lambda^{s}, \partial_{x} V\right] \partial_{x}^{2} u, \Lambda^{s} u\right)+2 \mu\left(\partial_{x} V \Lambda^{s} \partial_{x}^{2} u, \Lambda^{s} u\right) \\
& =2 \mu\left(\left[\Lambda^{s}, \partial_{x} V\right] \partial_{x}^{2} u, \Lambda^{s} u\right)-2 \mu\left(\partial_{x} V \Lambda^{s} \partial_{x} u, \Lambda^{s} \partial_{x} u\right)-2 \mu\left(\partial_{x}^{2} V \Lambda^{s} u, \Lambda^{s} \partial_{x} u\right) \\
& \lesssim \mu\left(\left|\partial_{x}^{2} V\right|_{H^{s-1}}\left|\partial_{x}^{2} u\right|_{H^{s-1}}|u|_{H^{s}}+\left|\partial_{x} V\right|_{H^{s} 0}\left|\partial_{x} u\right|_{H^{s}}^{2}+\left|\partial_{x}^{2} V\right|_{H^{s}}\left|\partial_{x} u\right|_{H^{s}}|u|_{H^{s}}\right) .
\end{aligned}
$$

- Similarly we have for all $s \geq s_{0}$, and $s_{0}>\frac{1}{2}$

$$
\begin{aligned}
a\left(\Lambda^{s}\left(h^{\prime}(\bar{u}) \partial_{x}^{2} u\right), \Lambda^{s} u\right) & =a\left(\left[\Lambda^{s}, h^{\prime}(\bar{u})\right] \partial_{x}^{2} u, \Lambda^{s} u\right)+k\left(h^{\prime}(\bar{u}) \Lambda^{s} \partial_{x} u, \Lambda^{s} \partial_{x} u\right)+k\left(\partial_{x} h^{\prime}(\bar{u}) \Lambda^{s} u, \Lambda^{s} \partial_{x} u\right) \\
& =a\left(\left[\Lambda^{s}, h^{\prime}(\bar{u})\right] \partial_{x}^{2} u, \Lambda^{s} u\right)+k\left(\left(h^{\prime}(\bar{u})-h^{\prime}(0)+h^{\prime}(0)\right) \Lambda^{s} \partial_{x} u, \Lambda^{s} \partial_{x} u\right)+k\left(\partial_{x} h^{\prime}(\bar{u}) \Lambda^{s} u, \Lambda^{s} \partial_{x} u\right) \\
& \lesssim\left|\partial_{x} h^{\prime}(\bar{u})\right|_{H^{s-1}}\left|\partial_{x}^{2} u\right|_{H^{s-1}}|u|_{H^{s}}+\left|h^{\prime}(\bar{u})-h^{\prime}(0)\right|_{H^{s_{0}}}\left|\partial_{x} u\right|_{H^{s}}^{2}+\left|\partial_{x} u\right|_{H^{s}}^{2}+\left|\partial_{x} h^{\prime}(\bar{u})\right|_{H^{s_{0}}}\left|\partial_{x} u\right|_{H^{s}}|u|_{H^{s}} \\
& \lesssim|\bar{u}|_{H^{s}}\left|\partial_{x}^{2} u\right|_{H^{s-1}}|u|_{H^{s}}+\left(|\bar{u}|_{H^{s}}+1\right)\left|\partial_{x} u\right|_{H^{s}}^{2}+|\bar{u}|_{H^{s_{0}+1}}\left|\partial_{x} u\right|_{H^{s}}|u|_{H^{s}}
\end{aligned}
$$

- Using the identity $\left[\Lambda^{s}, V\right] \partial_{x}^{3} u=\partial_{x}\left(\left[\Lambda^{s}, V\right] \partial_{x}^{2} u\right)-\left[\Lambda^{s}, \partial_{x} V\right] \partial_{x}^{2} u$ leads to

$$
\begin{aligned}
\mu\left(\left[\Lambda^{s}, V\right] \partial_{x}^{3} u, \Lambda^{s} u\right) & =\mu\left(\partial_{x}\left(\left[\Lambda^{s}, V\right] \partial_{x}^{2} u\right), \Lambda^{s} u\right)-\mu\left(\left[\Lambda^{s}, \partial_{x} V\right] \partial_{x}^{2} u, \Lambda^{s} u\right) \\
& =-\mu\left(\left[\Lambda^{s}, V\right] \partial_{x}^{2} u, \Lambda^{s} \partial_{x} u\right)-\mu\left(\left[\Lambda^{s}, \partial_{x} V\right] \partial_{x}^{2} u, \Lambda^{s} u\right) \\
& \lesssim \mu\left(\left|\partial_{x} V\right|_{H^{s-1}}\left|\partial_{x}^{2} u\right|_{H^{s-1}}\left|\partial_{x} u\right|_{H^{s}}+\left|\partial_{x}^{2} V\right|_{H^{s-1}}\left|\partial_{x}^{2} u\right|_{H^{s-1}}|u|_{H^{s}}\right)
\end{aligned}
$$

- An integrating by parts give us for all $s \geq s_{0}$, and $s_{0}>\frac{1}{2}$

$$
\frac{\mu}{2}\left(\partial_{x}^{3} V \Lambda^{s} u, \Lambda^{s} u\right)=-\mu\left(\partial_{x}^{2} V \Lambda^{s} \partial_{x} u, \Lambda^{s} u\right) \lesssim \mu\left|\partial_{x}^{2} V\right|_{H^{s}}|u|_{H^{s}}\left|\partial_{x} u\right|_{H^{s}}
$$

- Similarly we have for all $s \geq s_{0}$, and $s_{0}>\frac{1}{2}$

$$
\frac{3 \mu}{2}\left(\partial_{x}^{2} V \Lambda^{s} \partial_{x} u, \Lambda^{s} u\right) \lesssim \mu\left|\partial_{x}^{2} V\right|_{H^{s}}|u|_{H^{s}}\left|\partial_{x} u\right|_{H^{s}}
$$

- As before, we integrate by parts and using the product estimate to have for all $s \geq s_{0}$, and $s_{0}>\frac{1}{2}$ 


$$
\begin{aligned}
\frac{3 \mu}{2}\left(\partial_{x} V \partial_{x}^{2} \Lambda^{s} u, \Lambda^{s} u\right) & =-\frac{3 \mu}{2}\left(\partial_{x} V \partial_{x} \Lambda^{s} u, \Lambda^{s} \partial_{x} u\right)-\frac{3 \mu}{2}\left(\partial_{x}^{2} V \partial_{x}^{2} \Lambda^{s} u, \Lambda^{s} u\right) \\
& \lesssim \mu\left(\left|\partial_{x} V\right|_{H^{s} 0}|u|_{H^{s}}\left|\partial_{x} u\right|_{H^{s}}+\left|\partial_{x}^{2} V\right|_{H^{s}}|u|_{H^{s}}\left|\partial_{x} u\right|_{H^{s}}\right) .
\end{aligned}
$$

- Furthermore we have for all $s \geq s_{0}$, and $s_{0}>\frac{1}{2}$

$$
a\left(\left[\Lambda^{s}, \partial_{x} h^{\prime}(\bar{u})\right] \partial_{x} u, \Lambda^{s} u\right) \lesssim\left|\partial_{x}^{2} h^{\prime}(\bar{u})\right|_{H^{s-1}}|u|_{H^{s}}\left|\partial_{x} u\right|_{H^{s-1}} \lesssim|\bar{u}|_{H^{s+1}}|u|_{H^{s}}\left|\partial_{x} u\right|_{H^{s-1}} .
$$

- Also we have for all $s \geq s_{0}$, and $s_{0}>\frac{1}{2}$

$$
\frac{a}{2}\left(\partial_{x}^{2} h^{\prime}(\bar{u}) \Lambda^{s} u, \Lambda^{s} u\right) \lesssim\left|\partial_{x}^{2} h^{\prime}(\bar{u})\right|_{H^{s_{0}}}|u|_{H^{s}}^{2} \lesssim|\bar{u}|_{H^{s_{0}+2}}|u|_{H^{s}}^{2}
$$

Finally if we take $s_{0}=s-1$ we obtain

$$
\begin{aligned}
\frac{1}{2} e^{\lambda t} \partial_{t}\left(e^{-\lambda t} E^{s}(u)^{2}\right) & \lesssim|\bar{u}|_{H^{s+1}}|u|_{H^{s}}^{2}+\left(|\bar{u}|_{H^{s}}+1\right)|u|_{H^{s+1}}^{2}+\mu|V|_{H^{s+1}}|u|_{H^{s+1}}^{2}-\frac{\lambda}{2} E^{s}(u)^{2} \\
& \lesssim\left(|\bar{u}|_{H^{s+1}}+1\right)|u|_{H^{s+1}}^{2}+\mu|V|_{H^{s+1}}|u|_{H^{s+1}}^{2}-\frac{\lambda}{2} E^{s}(u)^{2}
\end{aligned}
$$

Thanks to the above inequality, one can choose

$$
\lambda=\lambda_{T}=2 \sup _{t \in[0, T]}\left(|\bar{u}|_{H^{s+1}}+1\right),
$$

one deduces

$$
e^{\lambda t} \partial_{t}\left(e^{-\lambda t} E^{s}(u)^{2}\right) \lesssim|V|_{H^{s+1}} E^{s}(u)^{2}
$$

Integrating this differential inequality yields

$$
\begin{aligned}
\forall t \in[0, T], E^{s}(u(t, x))^{2} & \lesssim e^{\lambda t} E^{s}\left(u_{0}(x)\right)^{2}+\int_{0}^{t} e^{\lambda(t-\zeta)}|V(\zeta, x)|_{H^{s+1}} E^{s}(u(\zeta, x))^{2} d \zeta \\
& \lesssim e^{\lambda T} E^{s}\left(u_{0}(x)\right)^{2}+\int_{0}^{t} e^{\lambda(t-\zeta)}|V(\zeta, x)|_{H^{s+1}} E^{s}(u(\zeta, x))^{2} d \zeta
\end{aligned}
$$

Then by estimate, one can deduce from estimate (16) that

$$
\begin{aligned}
\forall t \in[0, T], E^{s}(u(t, x))^{2} & \lesssim e^{\lambda T} E^{s}\left(u_{0}(x)\right)^{2}+\int_{0}^{t} e^{\lambda(t-\zeta)}|\bar{u}(\zeta, x)|_{H^{s}} E^{s}(u(\zeta, x))^{2} d \zeta \\
& \lesssim e^{\lambda T} E^{s}\left(u_{0}(x)\right)^{2}+\int_{0}^{t} e^{\lambda(t-\zeta)} E^{s}(u(\zeta, x))^{2} d \zeta
\end{aligned}
$$

next by using Gronwall Lemma we obtain

$$
E^{s}(u(t, x))^{2} \lesssim e^{\lambda t} E^{s}\left(u_{0}(x)\right)^{2} e^{\left(\int_{0}^{t} e^{\lambda(t-\eta)} d \eta\right)}=e^{\lambda t} E^{s}\left(u_{0}(x)\right)^{2} \exp \left(\frac{1}{\lambda}\left(e^{\lambda t}-1\right)\right) .
$$

Now, for the last estimate we have

$$
\begin{aligned}
E^{s-1}\left(\partial_{t} u\right)^{2}= & \left(\Lambda^{s-1} \partial_{t} u, \mathcal{T} \Lambda^{s-1} \partial_{t} u\right)=\left(\Lambda^{s-1} \partial_{t} u, \Lambda^{s-1} \mathcal{T} \partial_{t} u\right) \\
= & a\left(\Lambda^{s-1}\left(\partial_{x} h(\bar{u}) \partial_{x} u\right), \Lambda^{s-1} \partial_{t} u\right)+2 \mu\left(\Lambda^{s-1}\left(\partial_{x} V \partial_{x}^{2} u\right), \Lambda^{s-1} \partial_{t} u\right)+\mu\left(\Lambda^{s-1}\left(V \partial_{x}^{3} u\right), \Lambda^{s-1} \partial_{t} u\right) \\
& \quad+a\left(\Lambda^{s-1}\left(\partial_{x} h^{\prime}(\bar{u}) \partial_{x} u\right), \Lambda^{s-1} \partial_{t} u\right)+a\left(\Lambda^{s-1}\left(h^{\prime}(\bar{u}) \partial_{x}^{2} u\right), \Lambda^{s-1} \partial_{t} u\right),
\end{aligned}
$$

similarly, as in the estimate of $E^{s}(u(t, x))^{2}$ we apply a similar estimate in order to obtain 
Existence results for a monophasic compressible Darcy-Brinkman's flow in porous media

$$
E^{s-1}\left(\partial_{t} u\right)^{2} \lesssim\left(|\bar{u}|_{H^{s+1}}+1\right)|u|_{H^{s+1}}\left|\partial_{t} u\right|_{H^{s}}+\mu|V|_{H^{s+1}}|u|_{H^{s+1}}\left|\partial_{t} u\right|_{H^{s}}
$$

next, by using estimates (1) and Cauchy-schwarz inequalities we get

$E^{s-1}\left(\partial_{t} u\right)^{2} \lesssim\left((1+\mu)|\bar{u}|_{H^{s+1}}+1\right)|u|_{H^{s+1}}\left|\partial_{t} u\right|_{H^{s}} \lesssim \frac{1}{\gamma}\left((1+\mu)|\bar{u}|_{H^{s+1}}+1\right)|u|_{H^{s+1}}^{2}+\gamma\left((1+\mu)|\bar{u}|_{H^{s+1}}+1\right)\left|\partial_{t} u\right|_{H^{s}}^{2}$

since $\left(E^{s}\right)^{2}$ is an equivalent norm of the norm of space $H^{s+1}$ and by choosing $\gamma$ small enough we obtain

$$
E^{s-1}\left(\partial_{t} u\right)^{2} \leq C\left(\left|u_{0}\right|_{H^{s+1}}, \mu, \lambda\right), \forall t \in[0, T] .
$$

Theorem 1. Let $s_{0}>\frac{1}{2}, s \geq s_{0}+1$ and $u_{0} \in H^{s+1}$. Then there exist a positive $T$ such that for all $0 \leq t \leq T$, there exists a unique solution $(u, V)$ to $(6)$, in particular we have $u \in C\left([0, T] ; H^{s+1}\right) \cap C^{1}\left([0, T] ; H^{s}\right)$ and $V \in$ $C\left(0, T ; H^{s+2}\right)$.

Proof: We want to construct a sequence of approximate solution $\left(u_{n}, V_{n}\right)_{n \geq 0}$ by the iterative scheme

$$
u_{0}(t, x)=u_{0}(x), \text { and } \forall n \in \mathbb{N},\left\{\begin{aligned}
-\mu \partial_{x}^{2} V_{n+1}+V_{n+1} & =-a \partial_{x} h\left(u_{n}\right), \\
\partial_{t} u_{n+1}+B\left[V_{n+1}, u_{n}\right] \partial_{x} u_{n+1} & =0 \\
u_{n+1}(0, x) & =u_{0}(x)
\end{aligned}\right.
$$

where $B\left[V_{n+1}, u_{n}\right]=\mathcal{T}^{-1} A\left[V_{n+1}, u_{n}\right]$ and $A$ is defined as follows

$$
A\left[V_{n+1}, u_{n}\right] f=-a \partial_{x} h\left(u_{n}\right) f-2 \mu \partial_{x} V_{n+1} \partial_{x} f-\mu V_{n+1} \partial_{x}^{2} f-a \partial_{x} h^{\prime}\left(u_{n}\right) f-a h^{\prime}\left(u_{n}\right) \partial_{x} f .
$$

In order to prove the existence of a unique solution of system (6), we will prove that the iterative scheme (18) is convergent and the sequence $\left(u_{n}, V_{n}\right)_{n \geq 0}$ converges to $(u, V)$ where $(u, V)$ is the unique solution of system (6). By Proposition 1, we know that there is a unique solution $\left(u_{n+1}, V_{n+1}\right) \in C\left([0, T] ; H^{s+1}\right) \times L^{\infty}\left(0, T ; H^{s+2}\right)$ to (18) if $u_{n} \in C\left([0, T] ; H^{s+1}\right)$. Let $R>0$ be such that $E^{s}\left(u_{0}\right)^{2} \leq \frac{R}{2}$, it follows from proposition 1 , equation (16) that $u_{n+1}$ satisfies the following inequality

$$
\forall t \in[0, T], E^{s}\left(u_{n+1}(t, x)\right)^{2} \leq e^{\lambda T} E^{s}\left(u_{0}(x)\right)^{2}+\int_{0}^{t} e^{\lambda(t-\zeta)}\left|V_{n+1}(\zeta, x)\right|_{H^{s+1}} E^{s}\left(u_{n+1}(\zeta, x)\right)^{2} d \zeta,
$$

we suppose now that

$$
E^{s}\left(u_{n}(t, x)\right)^{2} \leq R
$$

then, using the fact that $\left|V_{n+1}\right|_{H^{s+1}} \leq E^{s}\left(u_{n}\right)$ yields to

$$
E^{s}\left(u_{n+1}(t, x)\right)^{2} \leq \frac{R}{2} e^{\lambda T}+\int_{0}^{t} R e^{\lambda(t-\zeta)} E^{s}\left(u_{n+1}(\zeta, x)\right)^{2} d \zeta
$$

next by using Gronwall Lemma we get

$$
E^{S}\left(u_{n+1}(t, x)\right)^{2} \leq \frac{R}{2} e^{\lambda T} \exp \left(\int_{0}^{t} R e^{\lambda(t-\zeta)} d \zeta\right)=\frac{R}{2} e^{\lambda T} \exp \left(\frac{R}{\lambda}\left(e^{\lambda t}-1\right)\right) .
$$

Hence, there is $T>0$ small enough such that

$$
\sup _{[0, T]} E^{s}\left(u_{n+1}(t, x)\right)^{2} \leq R .
$$

On the other hand, we will show that $\left(u_{n}\right)_{n \geq 0}$ is convergent, and to do this let us estimate $u_{n+1}-u_{n}$ 


$$
\begin{aligned}
\partial_{t}\left(E^{0}\left(u_{n+1}-u_{n}\right)^{2}\right) & =2\left(\mathcal{T} \partial_{t}\left(u_{n+1}-u_{n}\right), u_{n+1}-u_{n}\right) \\
& =-2\left(A\left[\left[V_{n+1}, u_{n}\right] \partial_{x}\left(u_{n+1}-u_{n}\right), u_{n+1}-u_{n}\right)-2\left(\left(A\left[V_{n+1}, u_{n}\right]-A\left[V_{n}, u_{n-1}\right]\right) \partial_{x} u_{n}, u_{n+1}-u_{n}\right) .\right.
\end{aligned}
$$

For the first term in the r.h.s of (20) it can be easily estimated for all $s_{0}>\frac{1}{2}$ as follow

$$
\begin{aligned}
-2\left(A\left[V_{n+1}, u_{n}\right] \partial_{x}\left(u_{n+1}-u_{n}\right), u_{n+1}-u_{n}\right) & \lesssim\left(\left|u_{n}\right|_{H^{s_{0}+1}}+1\right)\left|u_{n+1}-u_{n}\right|_{H^{1}}^{2}+\mu\left|V_{n+1}\right|_{H^{s_{0}+2}}\left|u_{n+1}-u_{n}\right|_{H^{1}}^{2} \\
& \lesssim\left((1+\mu)\left|u_{n}\right|_{H^{s_{0}+1}}+1\right)\left|u_{n+1}-u_{n}\right|_{H^{1}}^{2} .
\end{aligned}
$$

Also for the second term in the r.h.s of (20) it can be estimated by

$$
\begin{aligned}
-\left(\left(A\left[V_{n+1}, u_{n}\right]-A\left[V_{n}, u_{n-1}\right]\right) \partial_{x} u_{n}, u_{n+1}-u_{n}\right) \lesssim\left(\left|u_{n}\right|_{H^{s_{0}+2}}+1\right)\left|u_{n}-u_{n-1}\right|_{H^{1}}\left|u_{n+1}-u_{n}\right|_{H^{1}} \\
+\mu\left|u_{n}\right|_{H^{s_{0}+1}}\left|V_{n+1}-V_{n}\right|_{H^{1}}\left|u_{n+1}-u_{n}\right|_{H^{1}}
\end{aligned}
$$

and so on by the first equation of (18) and by estimate (13) we can obtain the following estimate

$$
\left|V_{n+1}-V_{n}\right|_{H^{s+1}} \lesssim\left|u_{n}-u_{n-1}\right|_{H^{s}}
$$

Next using estimate (19), we obtain

$$
\begin{aligned}
\partial_{t}\left(E^{0}\left(u_{n+1}-u_{n}\right)^{2}\right) & \leq C\left(\left|u_{0}\right|_{H^{s+1}}, \mu, R\right)\left|u_{n+1}-u_{n}\right|_{H^{1}}^{2}+C_{1}\left(\left|u_{0}\right|_{H^{s+1}}, \mu, R\right),\left|u_{n}-u_{n-1}\right|_{H^{1}}\left|u_{n+1}-u_{n}\right|_{H^{1}} \\
& \leq C\left(\left|u_{0}\right|_{H^{s+1}}, \mu, R\right)\left|u_{n+1}-u_{n}\right|_{H^{1}}^{2}+C_{1}\left(\left|u_{0}\right|_{H^{s+1}}, \mu, R\right),\left|u_{n}-u_{n-1}\right|_{H^{1}}^{2} \\
& \leq C\left(\left|u_{0}\right|_{H^{s+1}}, \mu, R\right) E^{0}\left(u_{n+1}-u_{n}\right)^{2}+C_{1}\left(\left|u_{0}\right|_{H^{s+1}}, \mu, R\right) E^{0}\left(u_{n}-u_{n-1}\right)^{2} \\
& \leq C\left(\left|u_{0}\right|_{H^{s+1}}, \mu, R\right) E^{0}\left(u_{n+1}-u_{n}\right)^{2}+C_{1}\left(\left|u_{0}\right|_{H^{s+1}}, \mu, R\right) \sup E^{0}\left(u_{n}-u_{n-1}\right)^{2} .
\end{aligned}
$$

Integrating with respect to $t$ give us

$$
\begin{aligned}
E^{0}\left(u_{n+1}-u_{n}\right)^{2} & \leq C\left(\left.u_{0}\right|_{H^{s+1}}, \mu, \lambda\right) \int_{0}^{t} E^{0}\left(u_{n+1}-u_{n}\right)^{2}+C_{1}\left(\left.u_{0}\right|_{H^{s+1}}, \mu, \lambda\right) \sup _{[0, T]}\left\{E^{0}\left(u_{n}-u_{n-1}\right)^{2}\right\} t \\
& \leq C\left(\left.u_{0}\right|_{H^{s+1}}, \mu, \lambda\right) \int_{0}^{t} E^{0}\left(u_{n+1}-u_{n}\right)^{2}+C_{1}\left(\left.u_{0}\right|_{H^{s+1}}, \mu, \lambda\right) \sup _{[0, T]}\left\{E^{0}\left(u_{n}-u_{n-1}\right)^{2}\right\} T .
\end{aligned}
$$

Furthermore, by using Gronwall Lemma we get

$$
\begin{array}{r}
E^{0}\left(u_{n+1}-u_{n}\right)^{2} \leq C_{1}\left(\left|u_{0}\right|_{H^{s+1}}, \mu, \lambda\right) \sup _{[0, T]}\left\{E^{0}\left(u_{n}-u_{n-1}\right)^{2}\right\} T \exp \left(C\left(\left|u_{0}\right|_{H^{s+1}}, \mu, \lambda\right) t\right) \\
\leq C_{1}\left(\left|u_{0}\right|_{H^{s+1}}, \mu, \lambda\right) \exp \left(C\left(\left|u_{0}\right|_{H^{s+1}}, \mu, \lambda\right) T\right) T \sup _{[0, T]}\left\{E^{0}\left(u_{n}-u_{n-1}\right)^{2}\right\} .
\end{array}
$$

This yield to

$$
\sup _{[0, T]}\left\{E^{0}\left(u_{n+1}-u_{n}\right)^{2}\right\} \leq\left(C_{1}\left(\left|u_{0}\right|_{H^{s+1}}, \mu, \lambda\right) \exp \left(C\left(\left|u_{0}\right|_{H^{s+1}}, \mu, \lambda\right) T\right) T\right)^{n} \sup _{[0, T]}\left\{E^{0}\left(u_{1}-u_{0}\right)^{2}\right\} .
$$

Finally, for $T>0$ small enough we have

$$
C_{1}\left(\left|u_{0}\right|_{H^{s+1}}, \mu, \lambda\right) \exp \left(C\left(\left|u_{0}\right|_{H^{s+1}}, \mu, \lambda\right) T\right) T<1 .
$$

Consequently, we conclude the existence of a time $T=T\left(\left|u_{0}\right|_{H^{s+1}}\right)$ and a function $u$ such that $u_{n}$ converge strongly to $u$ in $L^{\infty}\left(0, T ; H^{1}\right)$. On the other hand, we conclude from this convergence and by estimate (21) that there exist a function $V$ such that $V_{n}$ converges also strongly to $V$ in $L^{\infty}\left(0, T ; H^{2}\right)$. Since 
$\left(u_{n}, V_{n}\right)$ are bounded in $H^{s+1} \times H^{s+2}$ and according to the previous convergence we conclude that $(u, V) \in$ $L^{\infty}\left(0, T ; H^{s+1}\right) \times L^{\infty}\left(0, T ; H^{s+2}\right)$. Then we can pass to the limit in system $(18)$ when $n \rightarrow \infty$ to deduce that $(u, V)$ is a solution of system (6). Furthermore by interpolation results we obtain $\left|\left(u_{n}-u\right)(t, \cdot)\right|_{H^{s^{\prime}}} \rightarrow 0$ for all $s^{\prime}<s+1$ which gives us a strong convergence for $u_{n}$ and $V_{n}$ in higher space. Now we can prove $V \in C\left([0, T], H^{s+2}\right)$ and by classical argument see [23] we have that $u \in C\left([0, T], H^{s+1}\right)$. Finally the continuity of $\partial_{t} u$ can be obtained from first equation of (6).

\section{Study of Darcy-Brinkman's flow in $\mathbb{R}^{d}, d \geq 1$ with Bear hypothesis.}

In this section, we are interested in studying the system of Darcy-Brinkman with Bear hypothesis (see [6]) in a bounded domain multidimensional, which consists in neglecting the variation in density $\nabla \rho \cdot V$ in the direction of the flow and from this assumption one obtains our model.

First of all, let us recall that the system of Darcy-Brinkman with a source term is given by

$$
\left\{\begin{aligned}
\phi \partial_{t} \rho+V \cdot \nabla \rho+\rho \operatorname{div} V & =\rho f, \\
-\mu \Delta V+V & =-a \nabla p .
\end{aligned}\right.
$$

where $a=\frac{k}{\tilde{\mu}} \mu=\frac{v k}{\tilde{\mu}}$, with $k$ is the permeability coefficient, $\tilde{\mu}$ is the Darcy viscosity and $v$ is the Brinkman viscosity. Then by using Bear hypothesis $V \cdot \nabla \rho \lll 1$ and after applying the Helmholtz operator $(-\mu \Delta+I)$ on the first equation of (22), we get

$$
\phi \partial_{t} u-\mu \phi \Delta \partial_{t} u-\operatorname{div}(a \nabla p)=g
$$

with $u=\ln (\rho(p))$ and $p=\rho^{-1}\left(e^{u}\right)$. In the following section we are interested in some particular case of fluid in order to have a relationship between the pressure and the density.

\subsection{Some particular cases of state law}

Case 1: Ideal gas. In this case the relationship between the density and the pressure is given by

$$
p=p_{0} \rho^{\gamma}=p_{0} e^{\gamma u}
$$

hence

$$
\nabla p=\gamma p_{0} e^{\gamma u} \nabla u
$$

with $\gamma$ is the polytropic exponent, its value in the two main cases covered by this state when applied to gas are: $\gamma=1$ for isothermal process, and $\gamma$ bigger than 1 for adiabatic process, as example, the air with normal temperature, $\gamma=1.405$ this value is obtained by the experimental data). In all cases we have $\gamma \geq 1$.

So for this state, equation (23) becomes

$$
\phi \partial_{t} u-\mu \phi \Delta \partial_{t} u-\gamma a p_{0} \operatorname{div}\left(e^{\gamma u} \nabla u\right)=g
$$

Case 2: Slightly compressible gas. The state law is given by

$$
\rho(p)=\rho_{0} \exp \left(\zeta\left(p-p_{0}\right)\right)
$$

thus, 


$$
p=\frac{1}{\zeta}\left(p_{0}+\ln \frac{\rho}{\rho_{0}}\right)
$$

hence

$$
\nabla p=\frac{1}{\zeta} \frac{\nabla \rho}{\rho}=\frac{1}{\zeta} \nabla u
$$

equation (23) becomes linear and reads to :

$$
\phi \partial_{t} u-\mu \phi \Delta \partial_{t} u-\frac{a}{\zeta} \operatorname{div}(\nabla u)=g
$$

Cas 3: Another form of slightly compressible gas. The equation of this form is given by

$$
\rho(p)=\rho_{0}\left(1+\gamma\left(p-p_{0}\right)\right)
$$

which leads to $p=\frac{\rho-\rho_{0}}{\gamma \rho_{0}}+p_{0}$, consequently $\nabla p=\frac{1}{\gamma \rho_{0}} e^{u} \nabla u$. Furthermore, equation (23) becomes :

$$
\phi \partial_{t} u-\mu \phi \Delta \partial_{t} u-\frac{a}{\gamma \rho_{0}} \operatorname{div}\left(e^{u} \nabla u\right)=g
$$

In the next section, we prove the existence of a solution of a generalized system covering these 3 cases and specify the regularity of this solution.

\subsection{A generalized system of the Darcy-Brinkman equation}

Let $\Omega$ be a bounded domain in $\mathbb{R}^{d}$, with Lipschitz boundary $\partial \Omega$ and we denote by $\eta$ the outward normal vector. We set $Q_{T}:=[0, T] \times \Omega$ where $T>0$ is a fixed time. Our system is given by

$$
\left\{\begin{aligned}
\partial_{t} u-\mu \Delta \partial_{t} u-\operatorname{div}(f(u) \nabla u) & =g, & & (t, x) \in Q_{T}, \\
\mu \nabla \partial_{t} u \cdot \eta+\nabla u \cdot \eta & =0, & & (t, x) \in[0, T] \times \partial \Omega, \\
u(0, x) & =u_{0}(x), & & x \in \Omega
\end{aligned}\right.
$$

where $g$ is a function in $L^{2}\left(0, T ; H^{-1}(\Omega)\right)$, and $f$ is a continuous positive function. The medium is considered isotropic and homogeneous, then, as before we can change the scale in time to consider $\phi=1$. Furthermore, we introduce the notion of weak solutions of (24) as below.

Definition 1 We say that $u: Q_{T} \rightarrow \mathbb{R}$, is a weak solution of system (24) if $u_{0} \in H^{1}(\Omega) \cap L^{\infty}(\Omega)$ and for all $T>0, u \in W^{1, \infty}\left(0, T ; H^{1}(\Omega)\right)$, such that for all $\varphi \in L^{2}\left(0, T ; H^{1}(\Omega)\right)$ we have

$$
\int_{0}^{T} \int_{\Omega} \partial_{t} u \varphi d t d x+\mu \int_{0}^{T} \int_{\Omega} \nabla \partial_{t} u \cdot \nabla \varphi d t d x+\int_{0}^{T} \int_{\Omega} f(u) \nabla u \cdot \nabla \varphi d t d x=\int_{0}^{T} \int_{\Omega} g \varphi d t d x
$$

and $u(0, x)=u_{0}(x)$ in $H^{1}(\Omega)$.

We state the existence theorem of weak solutions of the system (24) as follow.

Theorem 2. Under assumption $u_{0} \in H^{1}(\Omega) \cap L^{\infty}(\Omega)$, the initial value problem (24) has a solution $u$ in the sense of the definition 1.

In additional, we establish the maximum principle first, then we prove theorem 2. 
Proposition 2. (Maximum principle) Let $u$ the solution of system (24), if $g$ in $L^{\infty}\left(Q_{T}\right)$, and if $u_{0} \in H^{1}(\Omega) \cap$ $L^{\infty}(\Omega)$ then

$$
-\max \left(|g|_{L^{\infty}\left(Q_{T}\right)},\left|u_{0}\right|_{L^{\infty}(\Omega)}\right) \leq u \leq \max \left(|g|_{L^{\infty}\left(Q_{T}\right)},\left|u_{0}\right|_{L^{\infty}(\Omega)}\right) e^{T} .
$$

Let us start by proving Proposition 2.

Proof : We multiply the first equation of (24) by $e^{\alpha t}$ and $\alpha$ real to be chosen later

$$
\partial_{t}\left(e^{\alpha t}\right)=e^{\alpha t} \partial_{t} u+\alpha e^{\alpha t} u
$$

we obtain

$$
\partial_{t}\left(e^{\alpha t} u\right)-\alpha e^{\alpha t} u-\mu \Delta\left(\partial_{t}\left(e^{\alpha t} u\right)-\alpha e^{\alpha t} u\right)-\operatorname{div}\left(f(u) \nabla\left(e^{\alpha t} u\right)\right)=e^{\alpha t} g
$$

let $w=e^{\alpha t} u$, the above equation is rewritten as

$$
\partial_{t} w-\alpha w-\mu \Delta \partial_{t} w+\alpha \mu \Delta w-\operatorname{div}(f(u) \nabla w)=e^{\alpha t} g .
$$

Let $\alpha<0$, then we write $\alpha=-|\alpha|$ and let $\beta$ a constant to be determined,

$$
\partial_{t}(w-\beta)+|\alpha|(w-\beta)-\mu \Delta \partial_{t}(w-\beta)-|\alpha| \mu \Delta(w-\beta)-\operatorname{div}(f(u) \nabla(w-\beta))=e^{-|\alpha| t} g-|\alpha| \beta .
$$

Multiply the above equation by $(w-\beta)^{+}$and integrate on $\Omega$

$$
\begin{aligned}
\int_{\Omega} \frac{d}{d t}\left|(w-\beta)^{+}\right|^{2} d x+|\alpha| \int_{\Omega}\left|(w-\beta)^{+}\right|^{2} d x & +\mu \int_{\Omega} \frac{d}{d t}\left|\nabla(w-\beta)^{+}\right|^{2} d x+|\alpha| \mu \int_{\Omega}\left|\nabla(w-\beta)^{+}\right|^{2} d x \\
& +\int_{\Omega} f(u)\left|\nabla(w-\beta)^{+}\right|^{2} d x=\int_{\Omega}\left(e^{-|\alpha| t} g-|\alpha| \beta\right)(w-\beta)^{+} d x
\end{aligned}
$$

we choose the parameter $\beta \geq \max _{Q_{T}}\left(\frac{e^{-|\alpha| t}}{|\alpha|}|g|\right)$ to impose that the left hand side is negative. Precisely,

$$
e^{-|\alpha| t} g-|\alpha| \beta \leq 0 \Longleftrightarrow \beta \geq \frac{e^{-|\alpha| t}}{|\alpha|} g, \forall(t, x) \in Q_{T} .
$$

then, by integrating over $] 0, t[$ and choosing $\alpha=-1$ we get

$$
\int_{\Omega}\left|(w-\beta)^{+}\right|^{2} d x+\mu \int_{\Omega}\left|\nabla(w-\beta)^{+}\right|^{2} d x \leq \int_{\Omega}\left|\left(u_{0}-\beta\right)^{+}\right|^{2} d x+\mu \int_{\Omega}\left|\nabla\left(u_{0}-\beta\right)^{+}\right|^{2} d x,
$$

Finally, by choosing $\beta=\max \left(|g|_{L^{\infty}\left(Q_{T}\right)},\left|u_{0}\right|_{L^{\infty}(\Omega)}\right)$ we get $\left(u_{0}-\beta\right)^{+}=0$, consequently $w \leq \beta$.

Similarly, to show that the solution is bounded below, we take $-(w-\beta)^{-}$as test function and we choose $\beta=-\max \left(|g|_{L^{\infty}\left(Q_{T}\right)},\left|u_{0}\right|_{L^{\infty}(\Omega)}\right)$.

Proof of theorem 2: The proof of theorem 2 is done in two steps. The first step consists to study a regularized problem, next for second step consists to pass to the limit as $\varepsilon \rightarrow 0$.

Step 1: Let $\varepsilon>0$ fixed. We introduce the regularization $f_{\varepsilon}$ instead of $f$ in the system (24) which is given by $f_{\varepsilon}(u)=\frac{f(u)}{1+\varepsilon f(u)}$. Then, we obtain the following regularized system :

$$
\left\{\begin{aligned}
\partial_{t} u_{\varepsilon}-\mu \Delta \partial_{t} u_{\varepsilon}-\operatorname{div}\left(f_{\varepsilon}\left(u_{\varepsilon}\right) \nabla u_{\varepsilon}\right) & =g, & & (t, x) \in Q_{T}, \\
\mu \nabla \partial_{t} u_{\varepsilon} \cdot \eta+\nabla u_{\varepsilon} \cdot \eta & =0, & & (t, x) \in[0, T] \times \partial \Omega, \\
u_{\varepsilon}(0, x) & =u_{0}(x), & & x \in \Omega .
\end{aligned}\right.
$$


The proof of the existence of solution $u$ of system (26) is based on Schauder fixed point theorem, for that we build the following application $\mathcal{F}$ given by :

$$
\begin{aligned}
\mathcal{F}: L^{2}\left(0, T ; L^{2}(\Omega)\right) & \longrightarrow L^{2}\left(0, T ; L^{2}(\Omega)\right) \\
\bar{u}_{\varepsilon} & \longrightarrow \mathcal{F}\left(\bar{u}_{\varepsilon}\right)=u_{\varepsilon},
\end{aligned}
$$

where $u_{\varepsilon}$ is the solution of the following linear problem

$$
\int_{\Omega} \partial_{t} u_{\varepsilon} \varphi d x+\mu \int_{\Omega} \nabla \partial_{t} u_{\varepsilon} \cdot \nabla \varphi d x+\int_{\Omega} f_{\mathcal{E}}\left(\bar{u}_{\varepsilon}\right) \nabla u_{\varepsilon} \cdot \nabla \varphi d x=\int_{\Omega} g \varphi d x .
$$

for all $\varphi \in L^{2}\left(0, T ; H^{1}(\Omega)\right)$. The existence and uniqueness of this solution is obtained by using of the FaedoGalerkin method, which give $u_{\varepsilon} \in H^{1}\left(0, T ; H^{1}(\Omega)\right)$, see for example [17].

Lemma $1 \mathcal{F}$ is a continuous map from $L^{2}\left(0, T ; L^{2}(\Omega)\right)$ to $L^{2}\left(0, T ; L^{2}(\Omega)\right)$.

Proof : Let $\bar{u}_{\varepsilon}^{n}$ be a sequence in $L^{2}\left(0, T ; L^{2}(\Omega)\right)$ and $\bar{u}_{\varepsilon} \in L^{2}\left(0, T ; L^{2}(\Omega)\right)$ such that

$$
\bar{u}_{\varepsilon}^{n} \longrightarrow \bar{u}_{\varepsilon} \text { in } L^{2}\left(0, T ; L^{2}(\Omega)\right) \text { when } n \longrightarrow \infty .
$$

Let $u_{\varepsilon}^{n}=\mathcal{F}\left(\bar{u}_{\varepsilon}^{n}\right)$ and $u_{\varepsilon}=\mathcal{F}\left(\bar{u}_{\varepsilon}\right)$, the aim is to show

$$
u_{\varepsilon}^{n} \longrightarrow u_{\varepsilon} \text { in } L^{2}\left(0, T ; L^{2}(\Omega)\right) \text { when } n \longrightarrow \infty .
$$

First of all, for all $n \in \mathbb{N}$, we have $u_{\varepsilon}^{n}$ and $u_{\varepsilon}$ are solution of (27), then take the difference between the two corresponding equations of $u_{\varepsilon}^{n}$ and $u_{\varepsilon}$, in addition if we take $\varphi=u_{\varepsilon}^{n}-u_{\varepsilon}$ as a test function in (27) and integrate in time we get:

$$
\begin{aligned}
\frac{1}{2} \int_{0}^{t} \int_{\Omega} \frac{d}{d t}\left|u_{\varepsilon}^{n}-u_{\varepsilon}\right|^{2} d s d x+\frac{\mu}{2} \int_{0}^{t} \int_{\Omega} \frac{d}{d t}\left|\nabla\left(u_{\varepsilon}^{n}-u_{\varepsilon}\right)\right|^{2} d s d x+\int_{0}^{t} \int_{\Omega} f_{\varepsilon}\left(\bar{u}_{\varepsilon}^{n}\right)\left|\nabla\left(u_{\varepsilon}^{n}-u_{\varepsilon}\right)\right|^{2} d s d x \\
\quad+\int_{0}^{t} \int_{\Omega}\left(f_{\varepsilon}\left(\bar{u}_{\varepsilon}^{n}\right)-f_{\varepsilon}\left(\bar{u}_{\varepsilon}\right)\right) \nabla u_{\varepsilon} \cdot \nabla\left(u_{\varepsilon}^{n}-u_{\varepsilon}\right) d s d x=0 .
\end{aligned}
$$

Next, we estimate each term of (28). The first one of the left hand side is writing as follows, since the two sequence have the same initial condition

$$
\frac{1}{2} \int_{0}^{t} \int_{\Omega} \frac{d}{d t}\left(u_{\varepsilon}^{n}-u_{\varepsilon}\right)^{2} d s d x=\frac{1}{2}\left\|u_{\varepsilon}^{n}-u_{\varepsilon}\right\|_{L^{2}(\Omega)}^{2} .
$$

Similarly, the second term is equivalent to

$$
\frac{\mu}{2} \int_{0}^{t} \int_{\Omega} \frac{d}{d t}\left(\nabla\left(u_{\varepsilon}^{n}-u_{\varepsilon}\right)\right)^{2} d s d x=\frac{1}{2}\left\|\nabla\left(u_{\varepsilon}^{n}-u_{\varepsilon}\right)\right\|_{L^{2}(\Omega)}^{2} .
$$

Furthermore, the third term is nonnegative

$$
\int_{0}^{t} \int_{\Omega} f_{\varepsilon}\left(\bar{u}_{\varepsilon}^{n}\right)\left(\nabla\left(u_{\varepsilon}^{n}-u_{\varepsilon}\right)\right)^{2} d s d x \geq 0 .
$$

Finally, for the last term by using Cauchy-Schwartz and Young inequalities

$$
\begin{aligned}
\int_{0}^{t} \int_{\Omega}\left(f_{\mathcal{\varepsilon}}\left(\bar{u}_{\varepsilon}^{n}\right)-f_{\mathcal{\varepsilon}}\left(\bar{u}_{\mathcal{\varepsilon}}\right)\right) \nabla u_{\varepsilon} \cdot \nabla\left(u_{\varepsilon}^{n}-u_{\varepsilon}\right) d s d x & \leq \\
& \frac{1}{2} \int_{0}^{t} \int_{\Omega}\left|\left(f_{\mathcal{\varepsilon}}\left(\bar{u}_{\varepsilon}^{n}\right)-f_{\varepsilon}\left(\bar{u}_{\mathcal{\varepsilon}}\right)\right) \nabla u_{\varepsilon}\right|^{2} d s d x+\frac{1}{2} \int_{0}^{t}\left\|\nabla\left(u_{\varepsilon}^{n}-u_{\varepsilon}\right)\right\|_{L^{2}(\Omega)}^{2} d s,
\end{aligned}
$$


but $f_{\varepsilon}\left(\bar{u}_{\varepsilon}^{n}\right)-f_{\varepsilon}\left(\bar{u}_{\varepsilon}\right) \rightarrow 0$ almost everywhere in $Q_{T}$, also we have $\left|\left(f_{\varepsilon}\left(\bar{u}_{\varepsilon}^{n}\right)-f_{\varepsilon}\left(\bar{u}_{\varepsilon}\right)\right) \nabla u_{\varepsilon}\right|^{2} \leq \frac{2}{\varepsilon}\left|\nabla u_{\varepsilon}\right|^{2} \in L^{1}\left(Q_{T}\right)$, then by Lebesgue dominated convergence theorem, then there exists a positive integer $n_{0}$ such that for all $n \geq n_{0}$ we have

$$
\int_{0}^{T} \int_{\Omega}\left|\left(f_{\varepsilon}\left(\bar{u}_{\varepsilon}^{n}\right)-f_{\mathcal{\varepsilon}}\left(\bar{u}_{\varepsilon}\right)\right) \nabla u_{\varepsilon}\right|^{2} \leq C_{n}
$$

where $C_{n}$ is positive constant tend to zero when $n \rightarrow+\infty$. Finally, by using Gronwall Lemma for $n \geq n_{0}$ we have

$$
\left\|u_{\varepsilon}^{n}-u_{\varepsilon}\right\|_{H^{1}(\Omega)}^{2} \leq C_{1}(\mu, T) C_{n}
$$

Consequently, we have $u_{\varepsilon}^{n} \longrightarrow u_{\varepsilon}$ strongly in $C^{0}\left(0, T ; L^{2}(\Omega)\right)$, and particularly in $L^{2}\left(0, T ; L^{2}(\Omega)\right)$.

Lemma $2 \mathcal{F}$ is an application from $L^{2}\left(0, T ; L^{2}(\Omega)\right)$ to $W^{1, \infty}\left(0, T ; H^{1}(\Omega)\right)$

Proof : Since $u_{\varepsilon} \in L^{2}\left(0, T ; H^{1}(\Omega)\right)$, one takes the solution $u_{\varepsilon}$ as a test function in the weak formulation (27), and gets that

$$
\frac{1}{2} \frac{d}{d t} \int_{\Omega}\left|u_{\varepsilon}\right|^{2} d x+\frac{\mu}{2} \frac{d}{d t} \int_{\Omega}\left|\nabla u_{\varepsilon}\right|^{2} d x+\int_{\Omega} f_{\varepsilon}\left(\bar{u}_{\varepsilon}\right)\left|\nabla u_{\varepsilon}\right|^{2} d x=\int_{\Omega} g u_{\varepsilon} d x .
$$

From the positivity of function $f_{\mathcal{E}}$, and thanks to Cauchy-Schwartz and Young inequalities, one can deduce by Gronwall Lemma that

$$
\left\|u_{\varepsilon}\right\|_{H^{1}} \leq C\left(\mu,\left\|u_{0}\right\|_{H^{1}},\|g\|_{L^{2}}\right) .
$$

Similarly, if we take $\partial_{t} u_{\varepsilon}$ as a test function in (27) we get

$$
\int_{\Omega}\left|u_{\varepsilon}\right|^{2} d x+\mu \int_{\Omega}\left|\nabla \partial_{t} u_{\varepsilon}\right|^{2} d x+\int_{\Omega} f_{\varepsilon}\left(\bar{u}_{\varepsilon}\right) \nabla u_{\varepsilon} \cdot \nabla \partial_{t} u_{\varepsilon} d x=\int_{\Omega} g \partial_{t} u_{\varepsilon} d x,
$$

by construction of $f_{\varepsilon}$ we have that $\left|f_{\varepsilon}\right| \leq \frac{1}{\varepsilon}$, then

$$
\int_{\Omega} f_{\varepsilon}\left(\bar{u}_{\varepsilon}\right) \nabla u_{\varepsilon} \cdot \nabla \partial_{t} u_{\varepsilon} d x \leq \frac{1}{2 \mu \varepsilon} \int_{\Omega}\left|\nabla u_{\varepsilon}\right|+\frac{\mu}{2} \int_{\Omega}\left|\nabla \partial_{t} u_{\varepsilon}\right|^{2} d x .
$$

Finally, by estimate (29) one cane deduce that

$$
\left\|\partial_{t} u_{\varepsilon}\right\|_{H^{1}} \leq C\left(\mu, \varepsilon,\left\|u_{0}\right\|_{H^{1}},\|g\|_{L^{2}}\right)
$$

We return to the proof of the theorem (2), since $\left.\mathcal{F}\left(L^{2}\left(0, T ; L^{2}(\Omega)\right)\right)\right) \subset W^{1, \infty}\left(0, T ; H^{1}(\Omega)\right)$ which is compact embedding in $L^{2}\left(0, T ; L^{2}(\Omega)\right)$. Then by Schauder's fixed point theorem there exist a solution $u_{\varepsilon} \in$ $W^{1, \infty}\left(0, T ; H^{1}(\Omega)\right)$ of problem (26) such that

$$
\int_{\Omega} \partial_{t} u_{\varepsilon} \varphi d x+\mu \int_{\Omega} \nabla \partial_{t} u_{\varepsilon} \cdot \nabla \varphi d x+\int_{\Omega} f_{\varepsilon}\left(u_{\varepsilon}\right) \nabla u_{\varepsilon} \cdot \nabla \varphi d x=\int_{\Omega} g \varphi d x, \forall \varphi \in L^{2}\left(0, T ; H^{1}(\Omega)\right) .
$$

Step 2: In this step we derive energy estimates on the sequence $\left(u_{\varepsilon}\right)_{\varepsilon}$ independently of $\varepsilon$ in order to pass to the limit. As $u_{\varepsilon}$ is a solution of (30), we take $\varphi=u_{\varepsilon}$ in (30), using the fact that $f_{\varepsilon}$ is nonnegative, and by Gronwall's Lemma we have:

$$
\left\|u_{\varepsilon}\right\|_{H^{1}(\Omega)}^{2} \leq C\left(\mu, g, u_{0}\right),
$$

where $C$ is a positive constant independent of $\varepsilon$. Similarly, we take $\varphi=\partial_{t} u_{\varepsilon}$ in (27) we get: 


$$
\left\|\partial_{t} u_{\varepsilon}\right\|_{L^{2}(\Omega)}^{2}+\mu|| \nabla \partial_{t} u_{\varepsilon} \|_{L^{2}(\Omega)}^{2}+\int_{\Omega} f_{\varepsilon}\left(u_{\varepsilon}\right) \nabla u_{\varepsilon} \cdot \nabla \partial_{t} u_{\varepsilon} d t d x=\int_{\Omega} g \partial_{t} u_{\varepsilon} d t d x
$$

Maximum principle in Proposition 2 depending only to the positiveness of $f_{\mathcal{E}}$, one can deduce

$$
-\max \left(|g|_{L^{\infty}\left(Q_{T}\right)},\left|u_{0}\right|_{L^{\infty}(\Omega)}\right) \leq u_{\varepsilon} \leq \max \left(|g|_{L^{\infty}\left(Q_{T}\right)},\left|u_{0}\right|_{L^{\infty}(\Omega)}\right) e^{T}
$$

because we only need to have $f$ is positive to apply this principle in Proposition 2 . Then by using this inequality we have

$$
\begin{aligned}
\int_{\Omega} f_{\varepsilon}\left(u_{\varepsilon}\right) \nabla u_{\varepsilon} \cdot \nabla \partial_{t} u_{\varepsilon} d t d x & \leq \frac{1}{4 \zeta}\left\|f\left(u_{\varepsilon}\right)\right\|_{L^{\infty}(\Omega)}\left\|\nabla u_{\varepsilon}\right\|_{L^{2}(\Omega)}^{2}+\zeta\left\|\nabla \partial_{t} u_{\varepsilon}\right\|_{L^{2}(\Omega)}^{2} \\
& \leq \frac{M}{4 \zeta}\left\|\nabla u_{\varepsilon}\right\|_{L^{2}(\Omega)}^{2}+\zeta\left\|\nabla \partial_{t} u_{\varepsilon}\right\|_{L^{2}(\Omega)}^{2}
\end{aligned}
$$

where $\zeta$ is a positive constant to be chosen later and $M=\max f\left(u_{\varepsilon}\right)$ where $u_{\varepsilon}$ satisfy inequality (33). Similarly using Cauchy-Schwartz and Young inequalities we obtain

$$
\int_{\Omega} g \partial_{t} u_{\varepsilon} d t d x \leq \frac{1}{4 \zeta}\|g\|_{L^{2} \Omega}^{2}+\zeta\left\|\partial_{t} u_{\varepsilon}\right\|_{L^{2}(\Omega)}^{2}
$$

Using estimate (31) and after choosing $\zeta$ small enough, we deduce from (32)

$$
\left\|\partial_{t} u_{\varepsilon}(t, .)\right\|_{L^{2}(\Omega)}+\mu\left\|\nabla \partial_{t} u_{\varepsilon}(t, .)\right\|_{L^{2}(\Omega)} \leq C\left(\mu, g, u_{0}\right), \text { for almost everywhere } t \in(0, T),
$$

where $C$ is a positive constant independent of $\varepsilon$. Consequently, the sequence $\left(u_{\varepsilon}\right)_{\varepsilon}$ is bounded in $W^{1, \infty}\left(0, T ; H^{1}(\Omega)\right)$, hence there exists a subsequence denoted $\left(u_{\varepsilon}\right)_{\varepsilon}$ converges strongly to $u$ in $\mathcal{C}^{0}\left(0, T ; L^{2}(\Omega)\right)$ by using AubinSimon Theorem [21]. Finally, as $f$ is continuous, then we can pass to the limit in (27) to obtain for all $\varphi \in L^{2}\left(0, T ; H^{1}(\Omega)\right)$ :

$$
\int_{\Omega} \partial_{t} u \varphi d t d x+\mu \int_{\Omega} \nabla \partial_{t} u \cdot \nabla \varphi d t d x+\int_{\Omega} f(u) \nabla u \cdot \nabla \varphi d t d x=\int_{\Omega} g \varphi d t d x
$$

and $u$ is the weak solution of system (24).

\section{References}

1. Adimurthi, MISHRA, S., and Gowda, G. V. Optimal entropy solutions for conservation laws with discontinuous flux-functions. Journal of Hyperbolic Differential Equations 2.04 (2005): 783-837.

2. Amiri, A., and K. Vafai. Analysis of dispersion effects and non-thermal equilibrium, non-Darcian, variable porosity incompressible flow through porous media. International Journal of Heat and Mass Transfer 37.6 (1994): 939-954.

3. Andreianov, B., Karlsen, K. H., and Risebro, N. H. A theory of $L^{1}$-dissipative solvers for scalar conservation laws with discontinuous flux. Archive for rational mechanics and analysis 201.1 (2011): 27-86.

4. Angirasa, D. Forced convective heat transfer in metallic fibrous materials. Transactions-American society of mechanical engineers journal of heat transfer 124.4 (2002): 739-745.

5. Auriault, J. Christian G. and Claude B. Filtration law in porous media with poor separation of scales. Transport in porous media 60.1 (2005): 89-108.

6. Bear, J. Dynamics of fluids in porous media. Courier Corporation, 2013.

7. Brinkman, H. C. A calculation of the viscous force exerted by a flowing fluid on a dense swarm of particles. Applied Scientific Research 1.1 (1949): 27-34.

8. Calmidi, V. V., and R. L. Mahajan. Forced convection in high porosity metal foams. Transactions-American society of mechanical engineers journal of heat transfer 122.3 (2000): 557-565.

9. Coclite, G., Mishra, S., and Risebro, N. Convergence of an Engquist-Osher scheme for a multi-dimensional triangular system of conservation laws. Mathematics of Computation 79.269 (2010): 71-94.

10. Coclite, G. M., and Risebro, N. H. Conservation laws with time dependent discontinuous coefficients. SIAM journal on mathematical analysis 36.4 (2005): 1293-1309. 
11. Dukhan, N. Analysis of Brinkman-extended Darcy flow in porous media and experimental verification using metal foam. Journal of fluids engineering 134.7 (2012): 071201.

12. Gimse, T., Risebro, N.H.: Solution of the Cauchy problem for a conservation law with discontinuous flux function. SIAM J.Math. Anal. 23(3), 635648 (1992)

13. Holden, H., Risebro, N.H.: Front Tracking for Hyperbolic Conservation Laws. Springer, Berlin (2011)

14. Israwi, Sa. Large time existence for 1D Green-Naghdi equations. Nonlinear Analysis: Theory, Methods and Applications 74.1 (2011) 81-93.

15. Kato, T., and Ponce, G. Commutator estimates and the euler and NavierStokes equations. Communications on Pure and Applied Mathematics, 41(7), (1988) 891-907. ISO 690

16. Karlsen, K.H., Risebro, N.H., Towers, J.D. $L^{1}$ Stability for entropy solutions of degenerate parabolic convection-diffusion equations with discontinuous coefficients. Skr. K. Nor. Vidensk. Selsk. 3, 149 (2003)

17. Ladyzhenskaia, O. A., Solonnikov, V. A., and Uraltseva, N. Linear and quasi-linear equations of parabolic type. Vol. 23. American Mathematical Soc., 1988.

18. Lannes, D. Sharp estimates for pseudo-differential operators with symbols of limited smoothness and commutators. Journal of Functional Analysis 232.2 (2006): 495-539.

19. Marusic-Paloka, E. Pazanin, I. and Marusic, S. Comparison between Darcy and Brinkman laws in a fracture. Applied mathematics and computation 218.14 (2012): 7538-7545.

20. Renken, K. J., and D. Poulikakos. Forced convection in channel filled with porous medium, including the effects of flow inertia, variable porosity, and Brinkman friction. ASME Journal of Heat transfer 109 (1987): 880-888.

21. Simon, J. Compact sets in the space $L^{p}(O, T ; B)$. Annali di Matematica pura ed applicata 146.1 (1986): 65-96.

22. S. Alinhac and P. Gérard. Opérateurs pseudo-différentiels et théorème de Nash-Moser. Inter-Editions et Editions du CNRS, Paris (1991).

23. Taylor, M. Partial differential equations II: Qualitative studies of linear equations. Vol. 116. Springer Science and Business Media, 2013. 